\title{
Simulation Analysis of Temporal Coding by Ionotropic Glutamate Receptors of Retinal OFF-type Bipolar Cells
}

\author{
Akito Ishihara* \\ Department of Mechanical and System Engineering, School of Engineering, Chukyo University, \\ 101-2 Yagoto-honmachi, Showa-ku, Nagoya, Aichi 466-8666, Japan
}

(Received August 2, 2017; accepted November 15, 2017)

Keywords: retinal OFF-type bipolar cells, ionotropic glutamate receptors, mathematical models, computer simulation, visual information processing

OFF-type bipolar cells (OFF-BCs) are interneurons connecting cone and retinal ganglion cells in the retina. In the mammalian retina, the cone signals are received by alpha-amino3-hydroxy-5-methyl-4-isoxazolepropionic acid (AMPA)/kainate-type ionotropic glutamate receptors (iGluRs) in OFF-BCs. The bipolar cells in the retina respond to continuous variations in light with a graded potential in an analogue manner. It is unclear what role the kinetics of the iGluRs play in the temporal coding and synaptic transmission from the cones to the OFFBCs. In this study, we analyzed the role of the kinetics of AMPA receptors (AMPARs) in transmitting visual information to the OFF-BCs by a computer simulation using developed mathematical models. We showed that the AMPARs modulated the frequency characteristics, which strongly depend on the baseline glutamate level. Our results also demonstrated that the response of OFF-BCs to the square-wave pulse of glutamate was similar to that of the ON-OFF type when the baseline glutamate level is around $0.5 \mathrm{mM}$, which enhanced temporal contrasts. The results suggest that the function would improve the sensitivities of the spatiotemporal contrast in collaboration with the local eye movement. This modeling study of retinal neurons would lead to the improvement and development of more effective stimulation for devices of retinal prostheses.

\section{Introduction}

The retina is a neural circuit that transmits processed visual information of scenes to the brain. ${ }^{(1,2)}$ Understanding the function of retinal visual information processing is not only important to clarify the mechanisms of vision in the brain, but is also one of the major challenges for applications in biological and medical engineering products, and for the development of intelligent information processing devices such as vision chips that emulate the spatial properties and light responses in the outer retina. ${ }^{(3)}$ In retinal prostheses, phosphenes are described by electrical stimulations to the inner retina of patients who have lost photoreceptor

*Corresponding author: e-mail: aishi@sist.chukyo-u.ac.jp

http://dx.doi.org/10.18494/SAM.2018.1720 
function from retinal dystrophies. Recently, some patients have recovered ultralow vision, which means that they can perceive large high-contrast shapes, recognize very large letters, and/or detect slow-moving objects. ${ }^{(4,5)}$ We consider that improvement in the spatial and temporal resolution of vision by retinal prostheses is necessary for the development of a device that converts a visual image into electrical stimulations that can induce similar activities like an intact retina. Even if the activities will incompletely mimic intact retinal activities, the revealed major function for visual information processing of the retina is essential for the development of the device. As the first step of this, it is important to understand neural transmission and visual information processing in synaptic connection from photoreceptors to bipolar cells in the retina because most of the devices for retinal prostheses stimulate bipolar cells and/or retinal ganglion cells (RGCs).

Bipolar cells are located in the main pathway of the retina, and receive inputs from photoreceptors in the outer plexiform layer (OPL) and send outputs to RGCs in the inner plexiform layer (IPL). In the OPL, several parallel channels originate, which extract different attributes from visual scenes. ${ }^{(6-8)}$ One of the channels is the ON and OFF channel. The OFF channel processes images that become visible by virtue of light intensity decrease; the ON channel processes in the reverse manner. The cones in all cases hyperpolarize in response to light and release glutamates as neurotransmitters in response to variation in the membrane potential in the synapse of OPL. In the dark, the cones persistently release glutamates at their resting potential. ${ }^{(9,10)}$ The membrane hyperpolarization in response to light suppresses the transmitter release. The bipolar cells that make contact with cones are divided into two major classes by their light response polarity. The OFF bipolar cells (OFF-BCs) have sign-conserving ionotropic glutamate receptors (iGluRs) and hyperpolarize in response to light in the same way as the cones. ${ }^{(11)}$ In contrast, the ON bipolar cells (ON-BCs) have sign-inverting metabotropic receptors and depolarize in response to light. ${ }^{(2)}$ In the IPL, the ON- and OFF-BCs in turn connect with the ON and OFF retinal ganglion cells, respectively, ${ }^{(12)}$ and the separated visual information from the retina is sent to the visual area of the brain.

In the past decade, the electrophysiological properties of the neurons in mammalian and primate retinas were revealed. Those studies demonstrated that there are two types of iGluRs of the non- $N$-methyl-D-aspartate (NMDA) type: alpha-amino-3-hydroxy-5-methyl-4isoxazolepropionic (AMPA) and kainate receptors (AMPAR/KAR). ${ }^{(10,13-21)}$ In the OFF-BCs of the mammalian retina, the glutamate-induced currents cause AMPARs to rapidly activate and then desensitize in response to steady glutamate application. ${ }^{(14,15,17,21)}$ On the other hand, that by the KARs has similar kinetics to AMPARs except for certain subtypes. ${ }^{(15,17-21)}$

In the central nervous system (CNS), such as in the cerebellum and cerebral cortex, it has been clarified that the AMPARs and KARs play roles in the learning and memory of neural mechanisms. ${ }^{(22)}$ Neurons in the CNS transmit information encoded by trains of action potentials to postsynaptic neurons by intermittent excitatory postsynaptic potentials via the AMPARs/KARs. The process of information transmission is digital. In contrast, the cones and bipolar cells in the retina respond to the continuous variation of light with a graded potential in an analogue manner. It is unclear what role the kinetics of the AMPARs/KARs play in the temporal coding and synaptic transmission to OFF-BCs. Because the bipolar cells 
are interconnected with other cells, not only in the OPL but also in the IPL, it is difficult to elucidate their role solely by electrophysiological methods.

In this study, the roles of the AMPARs of the OFF-BCs for temporal coding and visual information transmission mechanisms were analyzed by a computer simulation using a developed mathematical model of the glutamate receptor. We show that the frequency characteristics of OFF-BCs' responses to sinusoidal glutamate input strongly depended on the baseline glutamate concentration corresponding to the mean light level. Our results also demonstrated that the response of OFF-BCs to the square-wave pulse of glutamate is of the ON-OFF type when the baseline glutamate level is around $0.5 \mathrm{mM}$, which enhanced temporal contrasts. These results suggest that OFF-BCs transmit contrast information in conjunction with eye movement under different mean light intensities.

\section{Materials and Methods}

This section describes the outline of a constructed mathematical OFF-BC model, which was mounted on a new AMPAR model by adjusting the parameters of the CNS neurons in the published model. ${ }^{(26-30)}$ Numerical solutions and computer simulations using the developed model were obtained and run using MATLAB 2014a.

\subsection{Glutamate receptor model}

Glutamate application evokes the depolarization of the membrane potential and inward currents in OFF-BCs. The currents are induced by the AMPARs and/or the KARs. The kinetics of the current response that has been observed from the OFF-BCs of mammalian and primate retina shows an initial large transient followed by a sustained component, which is formed by the desensitization of the receptors ${ }^{(14,15,24)}$ [see also the response of our model in Fig. 1(b)]. The kinetics of the response to glutamate by AMPARs and most KARs is slightly different from other major features, and the structures of both receptors are similar. ${ }^{(25)}$ Therefore, this work analyzed the effects of glutamate-induced currents on the synaptic transmission mechanisms from the cones to OFF-BCs on the basis of an AMPAR model only.

Certain kinetic scheme models of AMPARs have been proposed and utilized to analyze the synaptic transmission and synaptic plasticity in CNS neurons and elsewhere. ${ }^{(26-30)}$ A model with such a detailed kinetics scheme in mammalian retinal neurons has not been published. Therefore, an AMPAR model of retinal OFF-BCs was constructed on the basis of Häusser's model. ${ }^{(26)}$ Figure 1(a) shows the kinetic scheme of the AMPAR model. The model assumes that an AMPAR has a total of nine states, namely, three closed states $\left(C_{0}-C_{2}\right)$, one open state $(O)$, and five desensitization states $\left(C_{3}-C_{7}\right)$. The kinetic rate equation can be written as

$$
\begin{gathered}
\frac{d C_{1}}{d t}=k_{C_{0} C_{1}}[\mathrm{Glu}] C_{0}+k_{C_{2} C_{1}} C_{2}+k_{C_{3} C_{1}} C_{3}-\left(k_{C_{1} C_{0}}+k_{C_{1} C_{2}}[\mathrm{Glu}]+k_{C_{1} C_{3}}\right) C_{1}, \\
\frac{d C_{2}}{d t}=k_{C_{1} C_{2}}[\mathrm{Glu}] C_{1}+k_{C_{4} C_{2}} C_{4}+k_{O C_{2}} O-\left(k_{C_{2} C_{1}}+k_{C_{2} C_{4}}+k_{C_{2} O}\right) C_{2},
\end{gathered}
$$




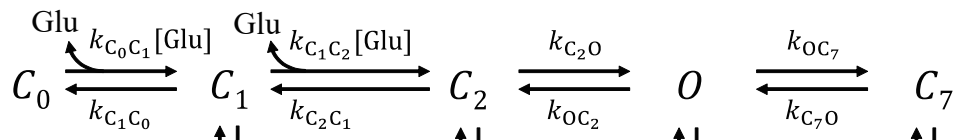

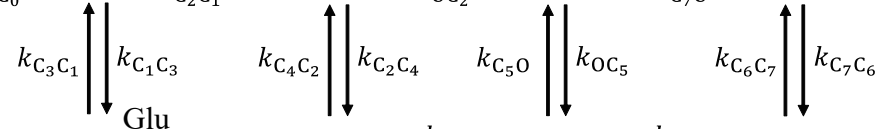

$$
\begin{aligned}
& C_{3} \underset{k_{\mathrm{C}_{4} \mathrm{C}_{3}}}{\stackrel{k_{\mathrm{C}_{3} \mathrm{C}_{4}}[\mathrm{Glu}]}{\rightleftarrows}} C_{4} \underset{k_{\mathrm{C}_{5} \mathrm{C}_{4}}}{\stackrel{k_{\mathrm{C}_{4} \mathrm{C}_{5}}}{\rightleftarrows}} C_{5} \underset{k_{\mathrm{C}_{6} \mathrm{C}_{5}}}{\stackrel{k_{\mathrm{C}_{5} \mathrm{C}_{6}}}{\rightleftarrows}} C_{6}
\end{aligned}
$$

(a)

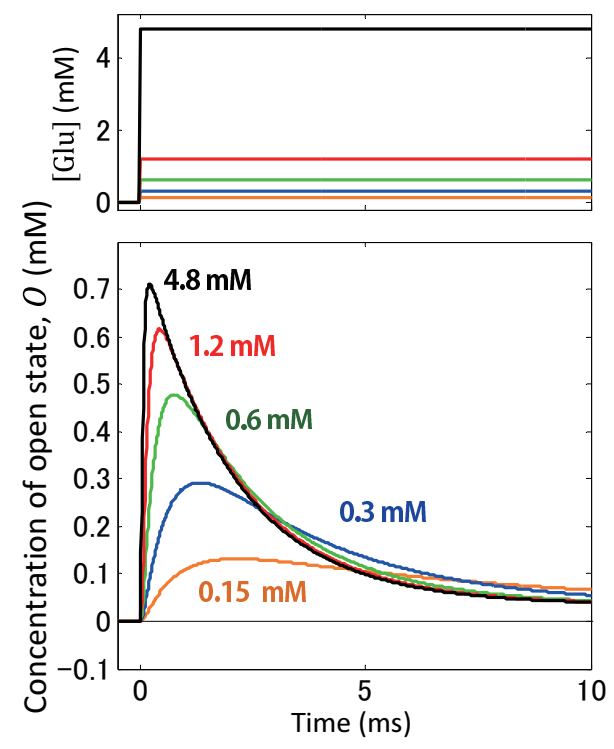

(b)

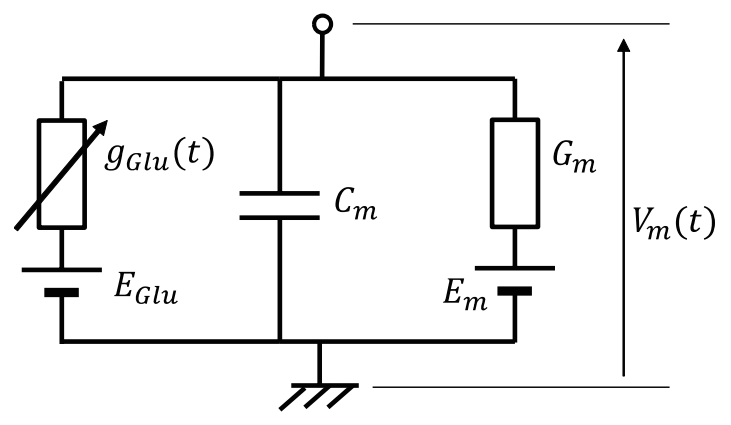

(c)

Fig. 1. (Color online) Simulated responses of the AMPAR model to glutamate application. (a) Kinetic scheme of the AMPAR model in OFF-BCs. (b) Responses of the open state (bottom) of the AMPAR model to glutamate concentrations (top) of 0.15 (orange), 0.3 (blue), 0.6 (green), 1.2 (red), and 4.8 (black) mM. (b) Equivalent circuit model of OFF-BCs.

$$
\begin{gathered}
\frac{d C_{3}}{d t}=k_{C_{1} C_{3}} C_{1}+k_{C_{4} C_{3}} C_{4}-\left(k_{C_{3} C_{1}}+k_{C_{3} C_{4}}[\mathrm{Glu}]\right) C_{3}, \\
\frac{d C_{4}}{d t}=k_{C_{2} C_{4}} C_{2}+k_{C_{3} C_{4}}[\mathrm{Glu}] C_{3}+k_{C_{5} C_{4}} C_{5}-\left(k_{C_{4} C_{2}}+k_{C_{4} C_{3}}+k_{C_{4} C_{5}}\right) C_{4}, \\
\frac{d C_{5}}{d t}=k_{C_{4} C_{5}} C_{4}+k_{C_{6} C_{5}} C_{6}+k_{O C_{5}} O-\left(k_{C_{5} C_{4}}+k_{C_{5} C_{6}}+k_{C_{5}} O\right) C_{5}, \\
\frac{d C_{6}}{d t}=k_{C_{5} C_{6}} C_{5}+k_{C_{7} C_{6}} C_{7}-\left(k_{C_{6} C_{5}}+k_{C_{6} C_{7}}\right) C_{6}, \\
\frac{d C_{7}}{d t}=k_{C_{6} C_{7}} C_{6}+k_{O C_{7}} O-\left(k_{C_{7} C_{6}}+k_{C_{7} O}\right) C_{7},
\end{gathered}
$$




$$
\frac{d O}{d t}=k_{C_{2} O} C_{2}+k_{C_{5} O} C_{5}+k_{C_{7} O} C_{7}-\left(k_{O C_{2}}+k_{O C_{7}}+k_{O C_{5}}\right) O
$$

where

$$
C_{0}=1-\left(C_{1}+C_{2}+C_{3}+C_{4}+C_{5}+C_{6}+C_{7}+O\right) \text {. }
$$

$C_{0}-C_{7}$, and $O$ are relative concentrations of receptors.

The time course of desensitization and the recovery from it in AMPAR responses to glutamate application in the OFF-BCs were faster than the responses of CNS neurons. ${ }^{(14,15)}$ The AMPAR model was thus modified from the original with a re-estimation of parameters using physiological data of the OFF-BCs in the ground squirrel retina. We focused on the following in our re-estimation.

(i) Dose response curve for high glutamate concentration application (half maximal concentration is around $340 \mu \mathrm{M})^{(15)}$

(ii) Time constants of initial rising phase $\left(\tau_{o n}<0.5 \mathrm{~ms}\right)$ to glutamate concentration jump and desensitization $\left(\tau_{D}=5-10 \mathrm{~ms}\right)$ in presence of $4.8 \mathrm{mM}$ glutamate $^{(15)}$

(iii) Time constants of deactivation to brief glutamate inputs $\left(\tau_{\text {off }}<2 \mathrm{~ms}\right)^{(15,28,29,31)}$

(iv) Time constants of recovery from desensitization $\left(\tau_{R}=18 \mathrm{~ms}\right)^{(14)}$

(v) The percentage of open state concentration in presence of $4.8 \mathrm{mM}$ glutamate $(<6 \%)^{(15)}$

Furthermore, the dose response curves of the sustained component of the current assumed that the concentration of the open state monotonically increases with the glutamate concentration, because there is no published data of AMPAR in OFF-BCs. The parameters of the model are summarized in Table 1.

Figure 1(b) shows an example of a simulated response of the AMPAR model to the sustained application of different concentrations of glutamate. This is a simulation of the experiment demonstrated by DeVries et al. ${ }^{(15)}$ Figure 1(b) shows the time course of the response as a function of the AMPAR open state concentration. With the application of glutamate [Fig. 1(b)], the concentration of the open state rapidly increased, forming transient components, and then

Table 1

Parameters of rate constants of the AMPAR model.

\begin{tabular}{llll}
\hline Parameters & Value (unit) & Parameters & Value (unit) \\
\hline$k_{C_{0} C_{1}}$ & $1.4 \times 10^{4}(1 / \mathrm{mM} / \mathrm{s})$ & $k_{C_{5} C_{4}}$ & $1.5 \times 10^{3}\left(\mathrm{~s}^{-1}\right)$ \\
$k_{C_{1} C_{0}}$ & $2.1 \times 10^{4}\left(\mathrm{~s}^{-1}\right)$ & $k_{C_{5} C_{6}}$ & $5.0 \times 10^{3}\left(\mathrm{~s}^{-1}\right)$ \\
$k_{C_{1} C_{2}}$ & $2.7 \times 10^{4}(1 / \mathrm{mM} / \mathrm{s})$ & $k_{C_{6} C_{7}}$ & $6.4 \times 10^{2}\left(\mathrm{~s}^{-1}\right)$ \\
$k_{C_{2} C_{1}}$ & $4.7 \times 10^{3}\left(\mathrm{~s}^{-1}\right)$ & $k_{C_{2} O}$ & $3.2 \times 10^{-1}\left(\mathrm{~s}^{-1}\right)$ \\
$k_{C_{1} C_{3}}$ & $4.2 \times 10^{2}\left(\mathrm{~s}^{-1}\right)$ & $k_{C_{7} C_{6}}$ & $1.9 \times 10^{3}\left(\mathrm{~s}^{-1}\right)$ \\
$k_{C_{3} C_{1}}$ & $7.8 \times 10^{1}\left(\mathrm{~s}^{-1}\right)$ & $k_{C_{2} O}$ & $1.7 \times 10^{4}\left(\mathrm{~s}^{-1}\right)$ \\
$k_{C_{3} C_{4}}$ & $2.7 \times 10^{4}(1 / \mathrm{mM} / \mathrm{s})$ & $k_{O C_{2}}$ & $3.7 \times 10^{3}\left(\mathrm{~s}^{-1}\right)$ \\
$k_{C_{4} C_{3}}$ & $6.6 \times 10^{2}\left(\mathrm{~s}^{-1}\right)$ & $k_{C_{5} O}$ & $6.9 \times 10^{-1}\left(\mathrm{~s}^{-1}\right)$ \\
$k_{C_{2} C_{4}}$ & $8.6 \times 10^{2}\left(\mathrm{~s}^{-1}\right)$ & $k_{O C_{5}}$ & $3.1 \times 10^{2}\left(\mathrm{~s}^{-1}\right)$ \\
$k_{C_{4} C_{2}}$ & $9.4 \times 10^{1}\left(\mathrm{~s}^{-1}\right)$ & $k_{C_{7} O}$ & $9.0 \times 10^{1}\left(\mathrm{~s}^{-1}\right)$ \\
$k_{C_{4} C_{5}}$ & $4.8 \times 10^{2}\left(\mathrm{~s}^{-1}\right)$ & $k_{O C_{7}}$ & $1.1 \times 10^{2}\left(\mathrm{~s}^{-1}\right)$ \\
\hline
\end{tabular}


slowly desensitized, forming a plateau, which represents a sustained component. The many features of the kinetics of AMPARs, such as those shown in Fig. 1 and particularly (i)-(v) above, were well reproduced by the proposed model.

\subsection{Membrane potential responses of OFF-BCs}

The membrane of retinal BCs has many ionic channels, and the membrane potentials that are evoked by a signal from a cone via glutamate receptors are also mediated by the nonlinear manner of their channels. The nonlinearity effect of their intrinsic ion channels can be analyzed with a model using ionic current mechanisms such as the Hodgkin-Huxley-type equations. ${ }^{(32)}$ Retinal BC models based on their ionic mechanisms have been proposed for the retina of some species: cyprinid fish, ${ }^{(33-35)}$ mice, ${ }^{(36)}$ and macaque monkeys. ${ }^{(37)}$ The models of the BCs were constructed on the basis of electrophysiological evidence from the cell. The electrophysiological data of membrane ionic properties for the modeling of the OFF-BCs of the mammalian retina have not yet been sufficiently clarified. In some cases, it is expected that the kinds of ionic channels, and their properties, in OFF cone BCs are also in rod BCs or other species' retina.

The purpose of this study was to elucidate the quantitative effects of AMPARs on the membrane potential responses and visual information processing of OFF-BCs. In order to analyze the relationship between kinetics of the AMPAR and membrane potential responses, the membrane of an OFF-BC was modeled simply using an isopotential cell ${ }^{(38)}$ with an equivalent circuit representation as shown in Fig. 1(c). From this model, one can write the following differential equation:

$$
C_{m} \frac{d V_{m}}{d t}=-\left[I_{G l u}+G_{m} \cdot\left(V_{m}-E_{m}\right)\right]
$$

where $V_{m}$ is the membrane potential, $C_{m}$ is the membrane capacitance, $G_{m}$ is the membrane conductance, $E_{m}$ is the resting potential of the OFF-BC, and $I_{G l u}$ is the glutamate-induced current. $I_{G l u}$ is given by the following equation:

$$
I_{G l u}=g_{G l u} \cdot\left(V_{m}-E_{G l u}\right),
$$

where $E_{G l u}$ is the reversal potential of the glutamate-induced current, and $g_{G l u}$ is the conductance of the glutamate-induced current and is equal to the product of the maximal conductance $\overline{G_{G l u}}$ and the concentration of the open state of the AMPAR model, $O$, as follows:

$$
g_{G l u}=\overline{G_{G l u}} \cdot O
$$

The $I-V$ relationship of the glutamate-induced current by the AMPAR of OFF-BCs indicates nonlinearity over $0 \mathrm{mV} .^{(20)}$ However, as the model was operated under $0 \mathrm{mV}$ in the simulation analysis, the voltage dependence of $I_{G l u}$ was simply described by Eq. (11).

The parameters of the membrane model of the OFF-BC [Fig. 1(c)] are summarized in Table 2. 
The $E_{G l u}$ value was a typical reversal potential of the AMPAR-mediated current, and $\overline{G_{G l u}}$ was estimated from the peak of the current response. ${ }^{(15)}$ The values of $G_{m}$ and $E_{m}$ were estimated assuming that the resting potential is around $-50 \mathrm{mV}^{(23)}$ under continuous $1.0 \mathrm{mM}$ glutamate application. The value of $C_{m}$ was estimated from the specific membrane capacitance of a typical neuron $\left(1.0 \mu \mathrm{F} / \mathrm{cm}^{2}\right)$ and the surface area of the body of OFF-BC. ${ }^{(14)}$ Initial conditions for the numerical simulation of responses are summarized in Table 3.

\section{Results}

\subsection{Responses of membrane potential of OFF-BC to glutamate pulses}

For the analysis of the light response mechanisms of OFF-BCs, the responses to different concentrations of glutamate in a square pulse were simulated using the model. As mentioned previously, glutamate is continuously released from a cone synapse in the dark and the glutamate concentration is decreased by the hyperpolarization of the cone that receives the light stimulus. The baseline concentration of glutamate in the dark varies in the range of $10^{-5}-10^{-2}$ M. ${ }^{(15)}$ To analyze the light-evoked membrane potential response, the glutamate responses were simulated under the baseline glutamate concentration, which in AMPARs desensitize almost completely.

Figure 2(a) shows the response of the glutamate-induced current $\left(I_{G l u}\right)$ and membrane potential $\left(V_{m}\right)$ to a square pulse of different glutamate concentrations of $100 \mathrm{~ms}$ width (from 0 to $100 \mathrm{~ms}$ ) with a baseline concentration of $1.0 \mathrm{mM}$. The $I_{G l u}$ [Fig. 2(a)] were slightly affected by the change in the lower glutamate concentration (from 1.0 to $0.01-0.1 \mathrm{mM}$ ); in contrast, there was no response to increases in concentration (from 1.0 to $2.0-5.0 \mathrm{mM}$ ). The

Table 2

Parameters of the OFF-BC model.

\begin{tabular}{lc}
\hline Parameters & Value (unit) \\
\hline$\overline{G_{G l u}}$ & $41(\mathrm{nS})$ \\
$E_{G l u}$ & $0(\mathrm{mV})$ \\
$C_{m}$ & $3.8(\mathrm{pF})$ \\
$G_{m}$ & $1.45(\mathrm{nS})$ \\
$E_{m}$ & $-100(\mathrm{mV})$ \\
\hline
\end{tabular}

Table 3

Initial condition of simulations.

\begin{tabular}{llllllllll}
\hline $\begin{array}{c}{[\mathrm{Glu}]_{0}} \\
(\mathrm{mM})\end{array}$ & $\begin{array}{c}C_{1} \\
(\mathrm{mM})\end{array}$ & $\begin{array}{c}C_{2} \\
(\mathrm{mM})\end{array}$ & $\begin{array}{c}C_{3} \\
(\mathrm{mM})\end{array}$ & $\begin{array}{c}C_{4} \\
(\mathrm{mM})\end{array}$ & $\begin{array}{c}C_{5} \\
(\mathrm{mM})\end{array}$ & $\begin{array}{c}C_{6} \\
(\mathrm{mM})\end{array}$ & $\begin{array}{c}C_{7} \\
(\mathrm{mM})\end{array}$ & $\begin{array}{c}O \\
(\mathrm{mM})\end{array}$ & $\begin{array}{c}V_{m} \\
(\mathrm{mV})\end{array}$ \\
\hline 0 & 0 & 0 & 0 & 0 & 0 & 0 & 0 & 0 & - \\
1.0 & 0.001 & 0.008 & 0.006 & 0.23 & 0.081 & 0.64 & 0.002 & 0.034 & -51.1 \\
0.4 & 0.003 & 0.008 & 0.014 & 0.22 & 0.080 & 0.63 & 0.002 & 0.033 & -51.9 \\
0.2 & 0.006 & 0.007 & 0.026 & 0.21 & 0.076 & 0.60 & 0.002 & 0.031 & -53.5 \\
0.1 & 0.011 & 0.006 & 0.044 & 0.18 & 0.065 & 0.51 & 0.002 & 0.026 & -57.9 \\
0.05 & 0.004 & 0.004 & 0.059 & 0.12 & 0.043 & 0.34 & 0.001 & 0.017 & -67.8 \\
\hline
\end{tabular}

$[\mathrm{Glu}]_{0}$ is baseline level or median of concentration of glutamate. 
membrane potentials were hyperpolarized by low-glutamate-concentration pulses from -50 $\mathrm{mV}$ to approximately $-90 \mathrm{mV}$. When the pulse terminated at $100 \mathrm{~ms}$, the membrane evoked depolarization, whose amplitudes were larger for the lower glutamate concentration.

Figure 2(b) shows the peak of the sustained component and transient component after pulse termination against glutamate concentration. For comparison, the dose response curve (dashed line), which is derived from Fig. 1(b), is also plotted in this figure. The sustained component of the responses (open circles) decreased with increased glutamate concentration and was proportional to the logarithm of the glutamate concentration in the range of $0.03-0.1 \mathrm{mM}$, while the transient component increased. The glutamate concentration dependence of these components was quite different from the dose response curve (dashed line).

We investigated the cause of the $\mathrm{ON}$ response properties by analyzing the responses of the AMPAR states. Figure 3 shows the responses of six states in the AMPAR model, which exhibit a large change with the application of the same simulation as in Fig. 2. $C_{0}$ is a nonbinding state, $C_{3}-C_{6}$ are desensitization states, and $O$ is an open state. In the presence of $1.0 \mathrm{mM}$ glutamate under the initial condition, AMPAR was slightly opened because of the desensitization, and the summation of $C_{4}-C_{6}$ was near $1.0 \mathrm{mM}$. When the concentration was $0.01 \mathrm{mM}$ (solid lines), AMPAR was gradually deactivating $\left(C_{0}\right.$, top left) and the concentration of the desensitization state also declined $\left(C_{4}-C_{6}\right.$, middle and bottom left). For the relatively high concentration stimulation $(0.1 \mathrm{mM}$, dotted lines), the deactivation of AMPAR was smaller. These results
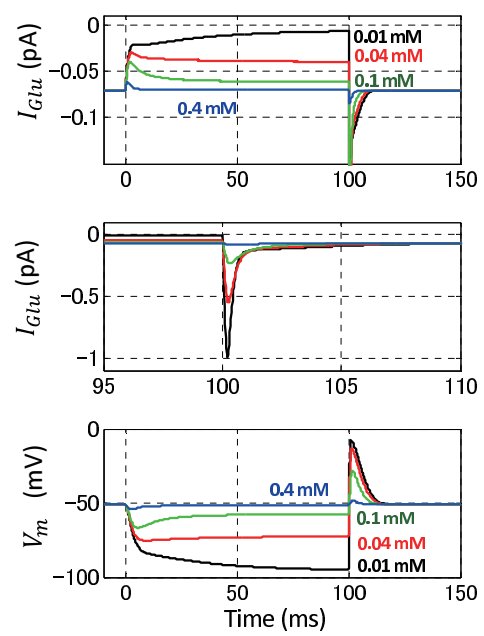

(a)

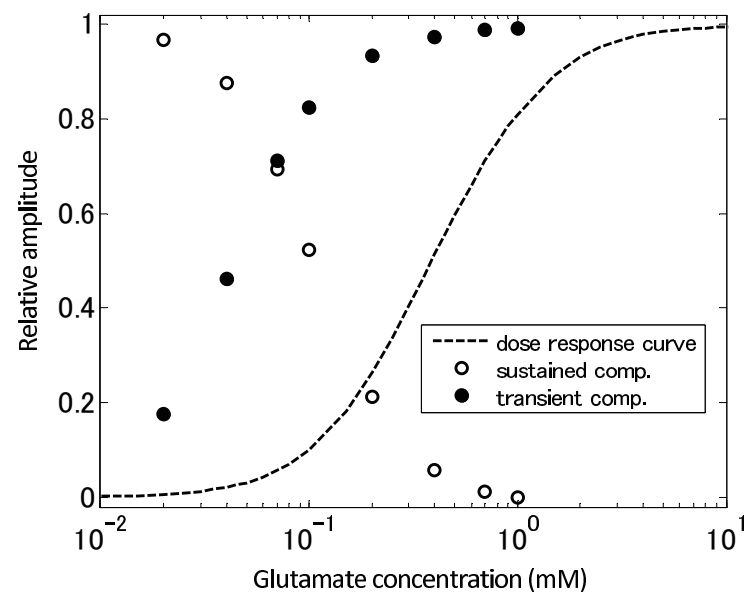

(b)

Fig. 2. (Color online) Responses to glutamate pulse with different concentrations. (a) Time course of $I_{G l u}$ (top and middle) and $V_{m}$ (bottom) responses to a pulse of different glutamate concentrations of $100 \mathrm{~ms}$ width. The middle figure shows an expanded view of the top figure. The glutamate concentration was changed from $1.0 \mathrm{mM}$ to 0.01 (black), 0.04 (red), 0.1 (green), $0.4 \mathrm{mM}$ (blue), and back to $1.0 \mathrm{mM}$. (b) Plot of the normalized responses versus glutamate concentration in (a). The peak of the sustained component during application of a glutamate pulse $(0-100 \mathrm{~ms}$, open circles) and the peak of the transient component after pulse termination (filled circles) are plotted against glutamate concentration. The dashed line indicates a dose-response curve of the peak relative amplitude of $I_{G l u}$ under the baseline glutamate concentration, which is 0 from the responses in Fig. 1(b). 

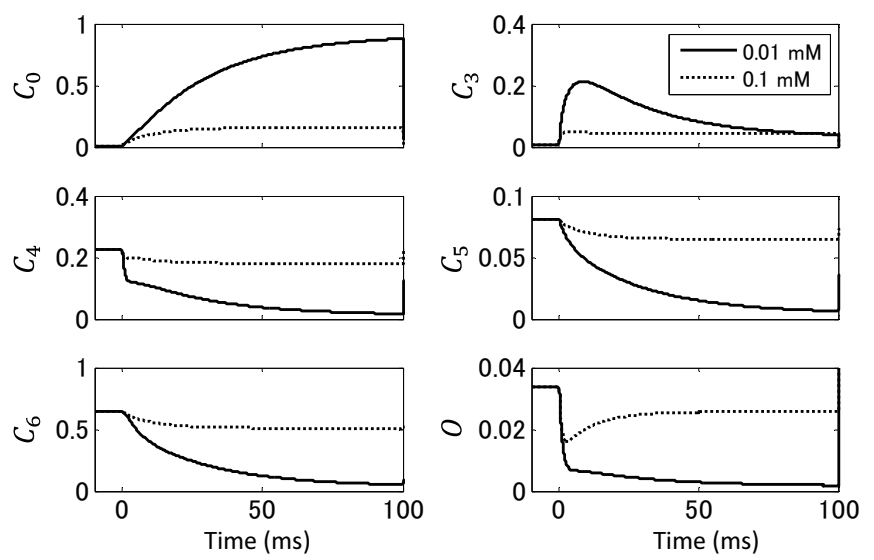

Fig. 3. Responses of AMPAR in the simulation in Fig. 2. The relative concentrations of six states that respond strongly are shown here although the AMPAR model has a total of nine states (see Sect. 2). $C_{0}$ is a nonbinding state, $C_{3}-C_{6}$ are desensitization states, and $O$ is an open state. All figures show the responses to low-concentration glutamate pulses, $0.1 \mathrm{mM}$ (solid line) and $0.01 \mathrm{mM}$ (dotted line).

suggest that the steady open rates of AMPAR and the dependence on the baseline concentration of glutamate will be determined by the rates of deactivation and desensitization.

\subsection{Responses of OFF-BC to sinusoidal glutamate inputs}

In the previous section, we showed the simulated responses to low glutamate concentration stimulus against baseline concentration. The responses correspond to responses to bright light against a dark background. The results showed that the time courses of the responses depend on the glutamate concentration. The abilities of temporal coding of the retinal cells have been checked using a sinusoidal stimulus. ${ }^{(19,23)}$ In this section, we also computationally analyze the effect of the kinetics of AMPAR on responses to sinusoidal glutamate input. It is expected that the baseline level of glutamate concentration around AMPARs in the cone synapse depends on mean light intensity. Therefore, we analyzed the relationship between frequency characteristics and the baseline concentration of glutamate input.

Figure 4(a) shows the responses of glutamate-induced current $\left(I_{G l u}\right)$ and membrane potential $\left(V_{m}\right)$ to a sinusoidal wave of glutamate with a median concentration of $0.1 \mathrm{mM}$, an amplitude of $0.01 \mathrm{mM}$, and a frequency of $10 \mathrm{~Hz}$ [Fig. 4(a) top]. Voltage responses [Fig. 4(a) bottom] exhibited distorted sinusoids, which were produced by $I_{G l u}$ [Fig. 4(a) middle] as described by the receptor's system of nonlinear equations. Figure 4(b) shows the frequency characteristics of glutamate-induced current [Fig. 4(b) top] and of membrane potential response [Fig. 4(b) bottom] to the stimulus with the same median and amplitude as the simulations in Fig. 4(a). These figures show the peak-to-peak amplitude of the responses against frequency. The responses of both $I_{G l u}$ and $V_{m}$ showed a band-pass filtering feature. The peak frequency of the voltage response was much lower than that of the glutamate-induced current because cell membranes have a low-pass filtering feature. 


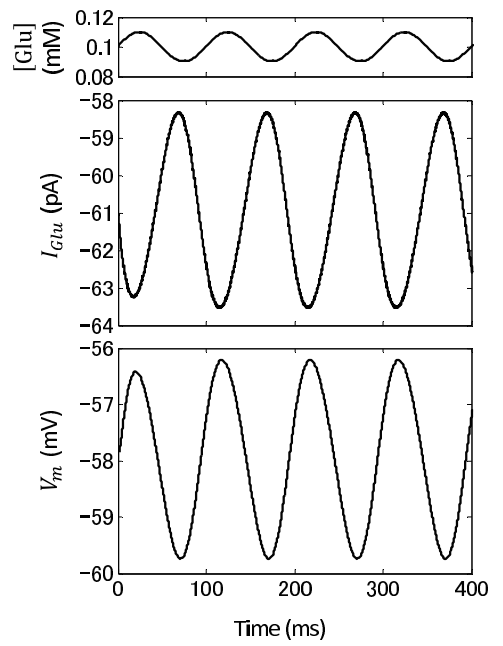

(a)
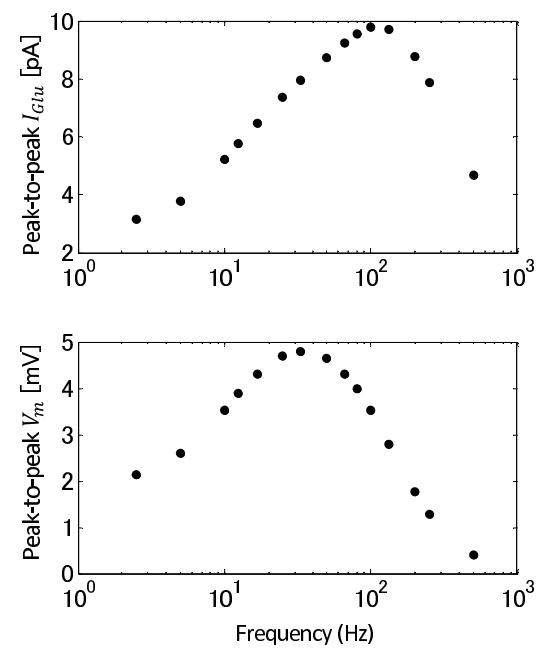

(b)

Fig. 4. Simulated responses of glutamate-induced current and membrane potential to sinusoidal glutamate inputs. (a) Time course of $I_{G l u}$ (middle) and $V_{m}$ (bottom) responses to a sinusoidal glutamate wave (top) with a median value of $0.1 \mathrm{mM}$, an amplitude of $0.01 \mathrm{mM}$, and a frequency of $10 \mathrm{~Hz}$. (b) Frequency characteristics of the peak-to-peak amplitude of $I_{G l u}$ (top) and $V_{m}$ (bottom).

The frequency characteristic of the OFF-BC strongly depends on the median of glutamate concentration. Figure 5 shows the relationship of the peak-to-peak amplitude of the membrane potential responses [Fig. 5(a)] with the frequency of sinusoidal glutamate inputs with median values of 0.05 (crosses), 0.1 (open squares), 0.2 (open circles), and $0.4 \mathrm{mM}$ (filled circles). The glutamate concentration has the amplitude of $10 \%$ of the median value, in order to adjust the condition so as to fluctuate at the same rate as that at the baseline concentration. Nevertheless, the voltage response increased as the mean concentration of glutamate decreased [Fig. 5(b) bottom]. The peak frequency was also decreased under the low glutamate concentration [Fig. 5(b) top]. There are two reasons why the amplitude under the low glutamate concentration was enhanced. First, the AMPARs were desensitized at a high glutamate concentration, as mentioned in the previous section. Second, the smaller the synaptic conductance relative to the membrane conductance, the larger the fluctuation of the membrane potential, because of the electrical property of the cell membrane, which is composed of parallel conductance.

These results suggest that the amplitude and frequency characteristics of the OFF-BC responses via AMPARs are strongly mediated by a baseline of the glutamate level, which is mainly controlled by the steady light intensity, and also suggest that the AMPAR desensitization mechanism is involved in OFF-BCs' responses under higher illuminance.

\subsection{Responses of OFF-BC to square pulse wave}

Regarding the frequency-response characteristics of OFF-BCs, as shown in the previous section, the OFF-BC and AMPAR system showed temporal band-pass filtering under a baseline 


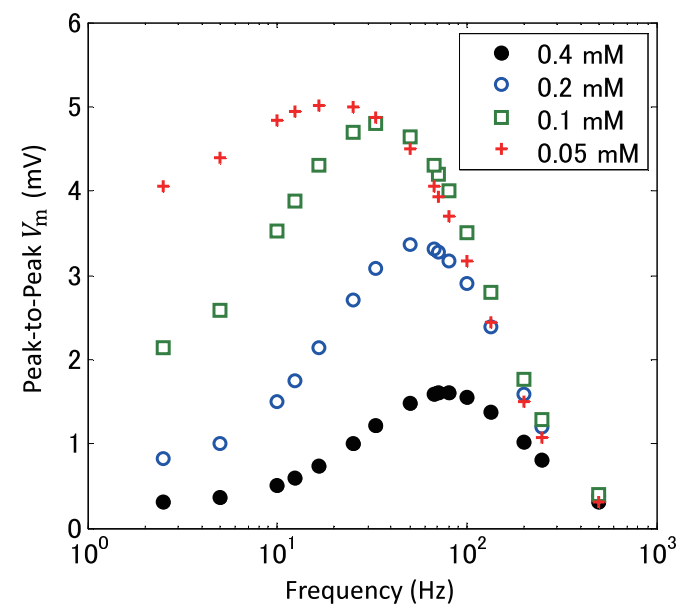

(a)
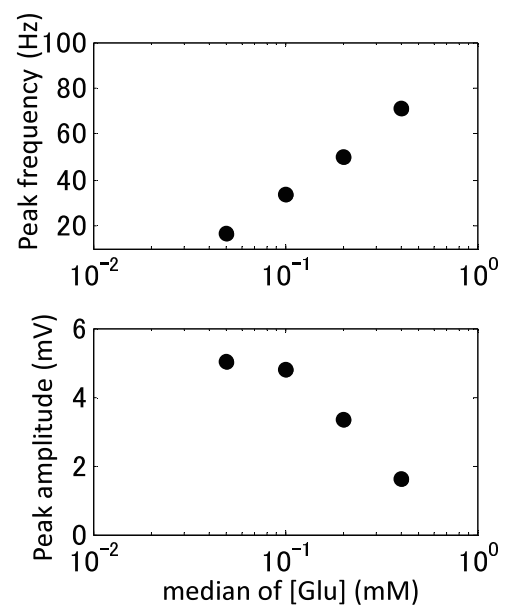

(b)

Fig. 5. (Color online) Frequency characteristics of the OFF-BC model depending on baseline glutamate concentration. (a) The peak-to-peak amplitude of $V_{m}$ was plotted versus the frequency of the sinusoidal stimulus with the amplitude of $10 \%$ of the median values of 0.05 (crosses), 0.1 (open squares), 0.2 (open circles), and 0.4 $\mathrm{mM}$ (filled circles). (b) Relationship between median glutamate concentration and peak frequency (top) and peak amplitude (bottom) from the frequency characteristics in (a).

glutamate level of $>0.1 \mathrm{mM}$. This means that the OFF-BC transmits the temporal variation in light at a middle-band frequency to the retinal ganglion cells under a relatively dark condition. Incidentally, our retina views a scene with irregularity because of local eye movement, i.e., microsaccades change the region of the scene sampled by the receptive field of the cells ${ }^{(39)}$ and the light information becomes a discrete time signal. Therefore, in this section, we examine the simulation of responses to a square pulse wave stimulus.

The responses to square pulse waves of glutamate concentration were different from the responses to the sinusoidal stimulus discussed in the previous section. Figure 6 shows waveforms of $I_{G l u}$ (middle) and membrane potential responses (bottom) after reaching a steady state. When the mean value of the square pulse stimulus was $0.4 \mathrm{mM}$ (Fig. 6 right column), transient components appeared in the ON and OFF phases with the pulse, and the membrane potential responses also had weakly transient components. In contrast, when the mean value was $0.2 \mathrm{mM}$ (Fig. 6 left column), the transient components were augmented by the low glutamate concentration. These results were reflected in the kinetics of AMPARs.

Figure 7(b) shows the frequency characteristics of the transient components (open circles) and sustained components (filled circles) of the response to the square pulse wave with a median value of $0.2 \mathrm{mM}$, which are the values immediately before the rising and falling edge of the pulse, as shown in Fig. 7(a). In the membrane potential responses [Fig. 7(b)], the sustained component increased as frequency increases from less than $100 \mathrm{~Hz}$ as well as responses to a sinusoidal stimulus. The results suggest that the OFF-BCs and AMPAR system would transmit temporal contrast augmented by transient components, and would perform just like the ONOFF cell under a high baseline level of glutamate, which corresponds to a mean light intensity. 

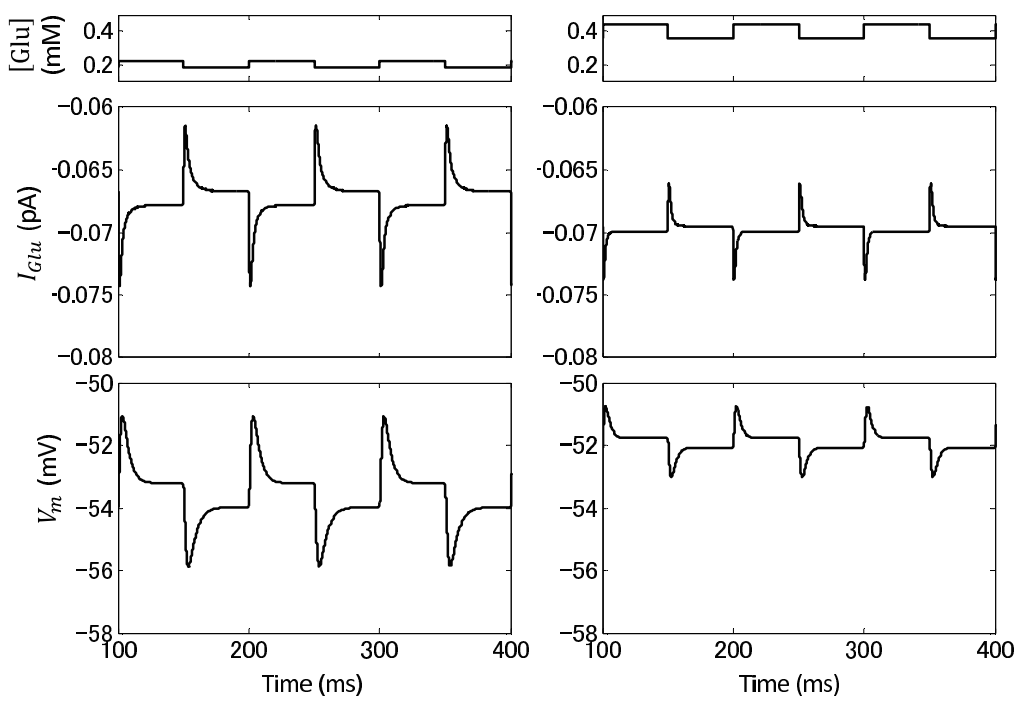

Fig. 6. Responses of glutamate-induced current and membrane potential to square pulse waves showing time course of $I_{G l u}$ (middle) and $V_{m}$ (bottom) responses to a square pulse wave (top) with median values of $0.2 \mathrm{mM}$ (left column) and $0.4 \mathrm{mM}$ (right column), amplitude of $10 \%$ of the median value, and frequency of $10 \mathrm{~Hz}$.

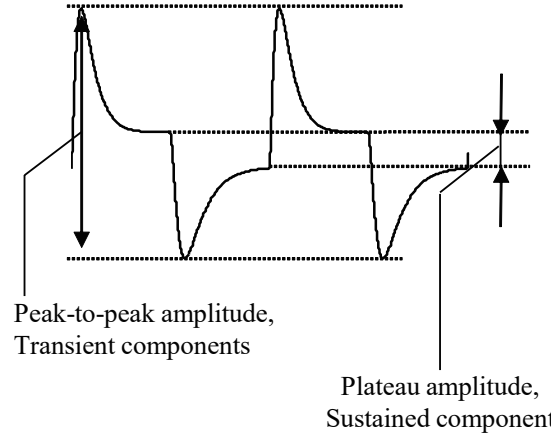

(a)

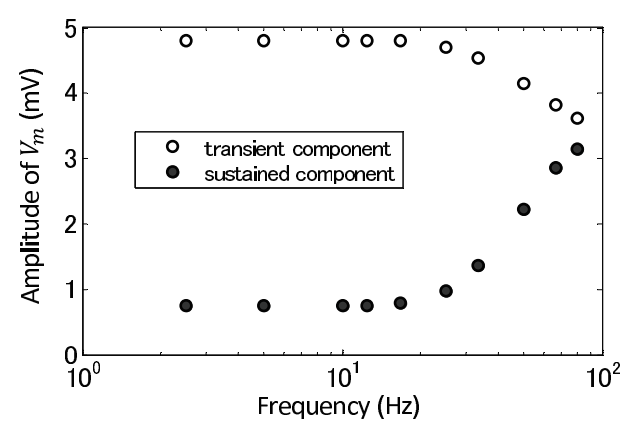

(b)

Fig. 7. Frequency characteristics of transient and sustained components in response to the square pulse waves with a median value of $0.2 \mathrm{mM}$. (a) The transient component is the peak-to-peak amplitude of responses, and the sustained component is the amplitude between ON and OFF plateau levels. (b) Plot of transient component (open circles) and sustained component (filled circles) of $V_{m}$ responses versus frequency of square pulse waves.

\section{Discussion}

Our study shows that the AMPAR system enhances temporal contrast, which depends on the baseline level of glutamate concentration and that this function is accomplished by the desensitization of AMPARs. The KARs in mammals have similar kinetics to AMPARs; ${ }^{(18,25)}$ however, it has been reported there are no, or weak, desensitization-type KARs in the rat ${ }^{(39)}$ and cat ${ }^{(13)}$ retina. If the glutamate-induced currents do not desensitize, the resting potential of BCs will be strongly depolarized in the dark by the currents. The resting potential of OFF- 
BCs is approximately $-50 \mathrm{mV} .^{(23)}$ Thus, it is necessary that the membrane conductance is very high when the currents exist. The larger membrane conductance will cause the decrease in the amplitude of the glutamate-induced current in response to light variations. It is considered that the desensitization of iGluRs plays a role in maintaining the low resting potential and in applying an appropriate amplitude of the responses of membrane potentials against the small variations of the cone signals.

Ichinose and Hellmer demonstrated that OFF-BCs in the mouse retina under photopic condition show a band-path filtering feature in response to sinusoidal light stimulus. ${ }^{(23)}$ The glutamate concentration around AMPARs varies as glutamates are released from cones in the cone pedicle. Therefore, we analyzed the relationship between the frequency characteristics of glutamate response and the median of glutamate concentration. In the results, OFFBCs responded to sinusoidal glutamate input with frequency characteristics similar to the electrophysiological results obtained by Ichinose and Hellmer. ${ }^{(23)}$ However, our results show that the peak amplitude and peak frequency of the characteristics depended on the baseline glutamate level corresponding to the mean light intensity (Fig. 5). The results suggest that the transmission in the synapse of cone-OFF-BCs is mediated by the temporal mean light intensity.

In the mouse retina, it has also been reported that OFF-BCs generate fast/transient and slow/sustained pathways by the differential expressions of KAR and AMPAR. ${ }^{(19)}$ Our results showed that the sustained component coded the glutamate concentration change in a band-pass filtering manner; in contrast, the transient component described the temporal contrast in a lowpass filtering manner. The band of the filter and the amplitude of the sustained and transient components were mediated by the kinetics of AMPAR, especially the recovery time course of desensitization. It is considered that the family of KAR with desensitization has a similar role to the OFF-BCs as a result of AMPARs. These results support the hypothesis that frequency channels of the retina begin with temporal coding in OFF-BCs in the first synapse of the visual system. ${ }^{(15,21,23)}$

We investigated the effect of eye movement on the OFF-BC and the AMPAR system using square pulse waves, as shown in Figs. 6 and 7. The glutamate concentration in the synaptic cleft between the cones and the OFF-BCs is rapidly changed by fast exocytosis, which has a time constant of $<5 \mathrm{~ms} .{ }^{(40,41)}$ We also simulated using stimuli output from the first-order lag system with a time constant of $5 \mathrm{~ms}$ using a square pulse train. The results showed that the amplitude of the ON-OFF phase decreased slightly (to approximately 70\%) compared with that in Fig. 6, but OFF-BCs exhibited similar responses to square waves (data not shown). The transient component appeared remarkably under the baseline glutamate concentration of $0.1-0.8 \mathrm{mM}$. The results suggest that AMPARs enhance fluctuations of light intensity by eye movement.

Our results show that OFF-BCs receive the middle frequency rather than low and high frequencies of cone signals and the characteristics were caused by AMPAR desensitization and the membrane circuit in OFF-BCs. In contrast, when cone signals are fluctuating, OFFBCs convert the amplitude of signals to the amplitude of the transient component of voltage responses, and operate as low-pass filters. It is known that lateral inhibition in the outer retina contributes to spatial contrast and contour enhancement. ${ }^{(1-3)}$ Our results suggest that the visual system composed the transmission from cones to OFF-BCs and eye movement carries out 
spatiotemporal differentiation similar to visual information processing as lateral inhibition, and the contrast enhancement depends on the mean background light intensity in the off pathway of the retina.

We thus consider that electrical signals obtained by the time derivative of light intensity will induce similar activities as in the intact retina when electrodes for the electrical stimulus are implanted near OFF-BCs in retinal prostheses devices. Many devices for retinal prostheses proposed today ${ }^{(4,5)}$ are placed at a certain position for stimulating inner retinal neurons, which are composed of BCs, amacrine cells, and RGCs. The stimulations suitable for those cells are unclear, but this study suggests that visual information at the AMPAR pathway from OFF-BCs to OFF-GCs will also be transmitted by the same manner. By maintaining the integration of retinal physiological evidence on mathematical models such as in our study, we would expect that the role AMPARs play in retinal information processing will be clarified, and the device for retinal prostheses will be improved by the development of preprocessing of visual image for their electrical stimulation.

\section{Conclusions}

In this study, we constructed a model of OFF-BCs, which was mounted on an AMPAR model by adjusting the parameters, and analyzed the roles of the AMPAR system in the visual information processing of OFF-BCs. The frequency characteristics of simulated responses to sinusoidal glutamate inputs depended on the baseline glutamate level that was caused by AMPAR desensitization and the membrane circuit in OFF-BCs. When the baseline glutamate level is around $0.5 \mathrm{mM}$, OFF-BCs played ON-OFF-type responses to pulse waves, which emphasized the temporal variation induced by AMPARs. The results suggest that the OFF-BC and AMPAR system transmits the enhancement of temporal contrast, and is modulated by the baseline glutamate level in the cone pedicle corresponding to mean light intensity. We expect that our results will lead to the improvement and development of a new stimulation method in the device for retinal prostheses.

\section{Acknowledgments}

This research was partially supported by the Ministry of Education, Culture, Sports, Science and Technology through a Grant-in-Aid for Scientific Research (C), 25330353.

\section{References}

1 A. Kaneko: J. Physiol. 207 (1970) 623.

2 F. S. Werblin and J. E. Dowling: J. Neurophysiol. 32 (1969) 339.

3 K. Shimonomura, S. Kameda, A. Iwata, and T. Yagi: IEEE Trans. Neural Networks 22 (2011) 1482.

4 A. Brandli, C. D. Luu, R. H. Guymer, and L. N. Ayton: Eye Brain 8 (2016) 15.

5 L. Bareket, A. Barriga-Rivera, M. P. Zapf, N. H. Lovell, and G. J. Suaning: J. Neural. Eng. 14 (2017) 045002.

6 H. Kolb: Webvision, eds. H. Kolb, R. Nelson, E. Fernandez, and B. Jones: http://webvision.med.utah.edu/ (accessed October 2017). 
7 R. W. Rodieck: The First Steps in Seeing (Sinauer, Sunderland, 1998).

8 P. H. Schiller: PNAS 107 (2010) 17087.

9 J. F. Ashmore and D. R. Copenhagen: J. Physiol. 340 (1983) 569.

10 S. H. DeVries and E. A. Schwartz: Nature 397 (1999) 157.

11 T. Saito and A. Kaneko: J. Gen. Physiol. 81 (1983) 589.

12 B. B. Boycott and H. Wässle: Eur. J. Neurosci. 3 (1991) 1069.

13 T. Sasaki and A. Kaneko: Vision Res. 36 (1996) 787.

14 S. H. DeVries: Neuron 28 (2000) 847.

15 S. H. DeVries, W. Li, and S. Saszik: Neuron 50 (2006) 735.

16 J. Pang, F. Gao, and S. M. Wu: Vision Res. 68 (2012) 48.

17 C. Puller, E. Ivanova, T. Euler, S. Haverkamp, and T. Schubert: Neurosci. 243 (2013) 136.

18 T. Euler, S. Haverkamp, T. Schubert, and T. Baden: Nat. Rev. Neurosci. 15 (2014) 507.

19 B. G. Borghuis, L. L. Looger, S. Tomita, and J. B. Demb: J. Neurosci. 43 (2014) 6128.

20 S. H. Lindstrom, D. G. Ryan, J. Shi, and S. H. DeVries: J. Physiol. 592 (2014) 1457.

21 T. P. Puthussery, K. A. Percival, S. Venkataramani, J. Gayet-Primo, U. Grünert, and W. R. Tayler: J. Neurosci. 34 (2014) 7611.

22 J. R. Whitlock, A. J. Heynen, M. G. Shuler, and M. F. Bear: Science 313 (2006) 1093.

23 T. Ichinose and C. B. Hellmer: J. Physiol. 596 (2016) 883.

24 D. Bowie: J. Physiol. 590 (2012) 49.

25 S. Zhu and E. Gouaux: Neuropharmacology 112 (2017) 11.

26 M. Häusser and A. Roth: J. Physiol. 501 (1997) 77.

27 J. I. Wadiche and C. E. Jahr: Neuron 32 (2001) 301.

28 A. Robert, S. N. Irizarry, T. E. Hughes, and J. R. Howe: J. Neurosci. 21 (2001) 5574.

29 A. Robert and J. R. Howe: J. Neurosci. 23 (2003) 847.

30 D. A. DiGregorio, J. S. Rothman, T. A. Nielsen, and R. A. Silver: J. Neurosci. 27 (2007) 8344.

31 G. B. Dawe, M. Musgaard, E. D. Andrews, B. A. Daniels, M. R. Aurousseau, P. C. Biggin, and D. Bowie: Nat. Struct. Mol. Biol. 20 (2013) 1054.

32 A. L. Hodgkin and A. F. Huxley: J. Physiol. 117 (1952) 500.

33 S. Usui, A. Ishihara, Y. Kamiyama, and H. Ishii: Vision Res. 36 (1996) 4069.

34 A. Ishihara, Y. Kamiyama, and S. Usui: Computational Neuroscience, ed. J. Bower (Plenum Press, New York, 1998) p. 203.

35 Y. Kamiyama, A. Ishihara, T. Aoyama, and S. Usui: Modeling in the Neurosciences, eds. G. N. Reeke, R. R. Poznanski, K. A. Lindsay, J. R. Rosenberg, and O. Sporns (CRC Press, Boca Raton, 2005) p. 313.

36 L. Oltedal, M. L. Veruki, and E. Hartveit: J. Physiol. 587 (2009) 829.

37 T. Puthussery, S. Venkataramani, J. Gayet-Primo, R. G. Smith, and W. R. Tayler: J. Neurosci. 33 (2013) 16045.

38 D. Johnston and S. M. Wu: Foundations of Cellular Neurophysiology (The MIT Press, London, 1995) Chap. 11.

39 T. Euller, H. Schneider, and H. Wässle: J. Neurosci. 16 (1996) 2934.

40 H. von Gersdorff, T. Sakaba, K. Berglund, and M. Tachibana: Neuron 21 (1998) 1177.

41 K. Rabl, L. Cadetti, and W. B. Thoreson: J. Neurosci. 25 (2005) 4633.

\section{About the Author}

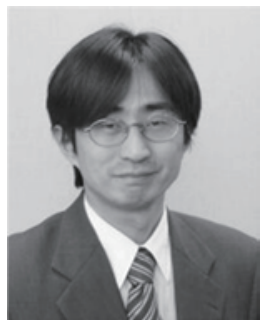

Akito Ishihara received his B.E., M.E., and Ph.D. degrees from Toyohashi University of Technology, Japan, in 1994, 1996, and 2000, respectively. From 2004 to 2008, he was an assistant professor, and from 2009 to 2012, he was an associate professor at Chukyo University, Japan. Since 2013, he has been a professor at Chukyo University. His research interests are in retinal visual information processing and information processing of neural circuits. 